\title{
KAHN Pierre \& MICHEL Youenn (dir.). Formation, transformations des savoirs scolaires. Histoires croisées des disciplines, $\mathrm{XIX}^{\mathrm{e}}-\mathrm{XX}^{\mathrm{e}}$ siècles
}

Nicole Tutiaux-Guillon

\section{(2) OpenEdition \\ 12 Journals}

Édition électronique

URL : http://journals.openedition.org/rfp/5003

DOI : $10.4000 / \mathrm{rfp} .5003$

ISSN : 2105-2913

Éditeur

ENS Éditions

\section{Édition imprimée}

Date de publication : 31 mars 2016

Pagination : 153-155

ISSN : 0556-7807

\section{Référence électronique}

Nicole Tutiaux-Guillon, « KAHN Pierre \& MICHEL Youenn (dir.). Formation, transformations des savoirs scolaires. Histoires croisées des disciplines, $x \mathrm{x}^{\mathrm{e}}-\mathrm{xx} \mathrm{e}^{\mathrm{e}}$ siècles », Revue française de pédagogie [En ligne], 194 | 2016, mis en ligne le 31 mars 2016, consulté le 05 janvier 2021. URL : http:// journals.openedition.org/rfp/5003; DOI : https://doi.org/10.4000/rfp.5003

Ce document a été généré automatiquement le 5 janvier 2021.

(c) tous droits réservés 


\title{
KAHN Pierre \& MICHEL Youenn (dir.). Formation, transformations des savoirs scolaires. Histoires croisées des disciplines, $\mathrm{XIX}^{\mathrm{e}}$ - $\mathrm{XX}^{\mathrm{e}}$ siècles
}

\author{
Nicole Tutiaux-Guillon
}

\section{RÉFÉRENCE}

KAHN Pierre \& MICHEL Youenn (dir.). Formation, transformations des savoirs scolaires. Histoires croisées des disciplines, $\mathrm{XIX}^{e}-\mathrm{XX} \mathrm{X}^{e}$ siècles. Caen : Presses universitaires de Caen, 2016, $318 \mathrm{p}$.

1 L'ouvrage fait suite à une recherche accréditée par l'Agence nationale de la recherche (ANR), mais sans exactement lui correspondre : si celle-ci s'encadrait dans l'intervalle 1945-années 1980, quatre chapitres ici concernent le XIX ou la première moitié du $\mathrm{xx}^{\mathrm{e}}$ siècle. La plupart des vingt-quatre auteurs s'inscrivent en histoire, en sociologie de l'éducation, en didactique et parfois aux croisements de ces domaines. Si on peut constater une juxtaposition des points de vue et des objets, l'ensemble porte sur les disciplines générales dans le primaire ou le secondaire, et sur certaines filières spécifiques, en France (sauf deux chapitres). Les deux premières parties se ciblent sur les savoirs ou les disciplines, la troisième aborde le rôle de certains acteurs et la dernière, "Réformes institutionnelles et savoirs scolaires ", envisage l'enseignement professionnel et l'enseignement spécialisé en s'intéressant parfois plutôt à la structure institutionnelle. Toutefois, les acteurs et les enjeux sociopolitiques sont loin d'être négligés dans les premières parties, et la question des contenus et de leurs fondements épistémologiques ou pédagogiques est prise en charge dans les analyses des jeux 
d'acteurs. On le voit, le titre de l'ouvrage n'est pas restrictif ; et celui-ci ne vise pas non plus à une quelconque exhaustivité : si l'éventail des disciplines est assez large, on relève aussi certaines absences. Ainsi les langues ne sont abordées qu'à travers les langues régionales, les sciences le sont peu, les mathématiques en France ne le sont pas, non plus que l'éducation civique ou les matières proprement techniques ou professionnelles. La présentation qui suit donne une idée des questions traitées et des disciplines représentées. L'introduction de l'ouvrage invite à d'autres lectures croisées, en particulier pour complexifier les approches d'histoire de l'enseignement par l'histoire des disciplines.

2 Trois chapitres se centrent sur le français : tous mettent en évidence les débats dont est l'objet cet enseignement, entre expression et littérature, du fait de sa charge culturelle et identitaire. À propos des programmes de français du lycée entre 1965 et 1981 et de la définition des épreuves du baccalauréat, Clémence Cardon-Quint éclaire les tensions entre les conceptions des belles-lettres et des finalités disciplinaires: la mise en programme qui en résulte a cherché avant tout à ne pas diviser davantage le corps professoral, aux dépens d'un renouvellement disciplinaire. Marie-France Bishop interroge la rénovation des contenus du français à l'école élémentaire à la même période: lieu de transposition de savoirs universitaires pluriels, mais aussi lieu de tensions entre acteurs. Anissa Belhadjin et Maryse Lopez montrent comment les changements du français dans l'enseignement professionnel dans la seconde moitié du $\mathrm{xx}^{\mathrm{e}}$ siècle reflètent les choix institutionnels concernant cette filière, entre relégation des élèves en échec, valorisation d'une voie spécifique et rapprochement avec le secondaire général ; au-delà de la discipline concernée, leurs interrogations rejoignent celles sur la culture scolaire.

Quatre chapitres traitent de savoirs scolaires référés aux sciences sociales; leurs auteurs montrent la complexité de la mise en programme et les effets, éventuellement contradictoires, des ancrages professionnels, politiques et épistémologiques. Micheline Roumégous analyse "l'invention de la géographie scolaire » en primaire entre 1850 et 1870, égratignant quelques idées reçues sur le rôle de la défaite de 1871 dans l'émergence de cette discipline. Elle souligne l'articulation entre nouvelle demande sociale de formation et d'ancrage dans les territoires national et local (de «territorialisation » dit-elle) et souci de renouveau pédagogique. Jean-Pierre Chevalier montre la conjonction entre pratiques de l'éveil et épistémologie de la géographie, conjonction qui a contribué à pérenniser des démarches actives inspirées des pratiques des géographes, soit dans les textes soit dans les faits, et légitimé la didactique de la géographie à ses débuts. Patricia Legris dissèque le jeu des acteurs institutionnels (groupe technique disciplinaire, inspection générale) et professionnels (Association des professeurs d'histoire et de géographie [APHG], syndicats) et le rôle de la consultation des enseignants dans l'écriture des programmes d'histoire de 1995. L'analyse du processus montre le primat des jeux de pouvoir sur les priorités didactiques. Élisabeth Chatel présente la façon dont, entre 1990 et 2010, les enseignants - ou plus exactement les «ténors de la profession» (p. 223) - ont infléchi la discipline sciences économiques et sociales, non seulement à partir de leurs choix politiques et scientifiques de contenus jugés pertinents (croisant les finalités disciplinaires) mais aussi, voire surtout, en se référant à des savoir-faire professionnels divergents. Ces contributions mettent bien en évidence comment les projets institutionnels sont contrebalancés par le rôle des enseignants, des finalités qu'ils assument et de leur épistémologie pratique. 
4 Si l'on tente un regroupement des chapitres relatifs aux enseignements mathématiques et scientifiques, il faut reconnaître leur grande hétérogénéité. Colette Le Lay présente un exemple des relations entre positionnement des scientifiques et des enseignants d'une part et épistémologie des savoirs scolarisés d'autre part, à travers l'exemple des évolutions de l'enseignement de "la science des astres » dans la première partie du $\mathrm{xIX}^{\mathrm{e}}$ siècle. Joël Lebeaume envisage la scolarisation progressive de l'enseignement ménager et de la technologie, en relation à la fois avec les missions de ces enseignements et les changements de contenus qui leur sont associés. Frédéric Charles, à partir des jeux d'alliances et d'opposition entre membres de la commission Lagarrigue, éclaire les relations entre physique, chimie, sciences de la vie et de la Terre et technologie. Livia Giacardi montre comment l'enseignement des mathématiques en Italie au début $\mathrm{du} \mathrm{xx}^{\mathrm{e}}$ siècle est un enjeu de démocratisation de l'enseignement. Elisabete Zardo Búrigo analyse comment dans les années 1960 une enseignante française, Lucienne Félix, a inspiré des enseignants brésiliens soucieux de rénover l'enseignement des mathématiques et de valoriser leur rôle.

5 Les autres chapitres sont relatifs chacun à une discipline scolaire singulière. Youenn Michel s'intéresse aux revendications, hésitations et compromis successifs de 1920 aux années 1980 qui ont permis la «constitution des langues régionales comme discipline scolaire» alors même que cet enseignement bouscule la culture républicaine universaliste et centralisatrice. Nathalie Dupont invite à une réflexion sur l'éducation artistique, à partir des « héritages de la période 1956-1969 », en dégageant les tensions entre le ministère de l'Éducation nationale, le ministère de la Culture et les mouvements d'éducation populaire, alors même que les visées de démocratisation culturelle sont partagées. Emmanuel Auvray et Yohann Fortune examinent, à travers les cas de la natation et de l'athlétisme, comment les formes d'évaluation de l'éducation physique, elles-mêmes liées aux conditions d'exercice du métier, ont structuré la discipline. Dominique Bret et Alix Le Moal abordent le corps des professeurs d'enseignement général de collège [PEGC] valence éducation physique et sportive (1968-1987) en soulignant comment l'évolution des contenus disciplinaires a conduit aussi à une évolution professionnelle qui a rapproché les PEGC des autres enseignants.

Certains chapitres ne touchent les savoirs qu'à travers d'autres questions. Laurent Gutierrez présente la Société française de pédagogie de 1919 à 1938 et les principes de rénovation qu'elle a portés, en particulier la mise en activité des élèves dans des démarches expérimentales. Il propose ainsi de nouveaux jalons sur la recherche d'une diffusion de pratiques fondées sur des acquis théoriques. Jean-Yves Seguy examine le cas des classes d'orientation sous le Front populaire ou plus exactement les procédures et les critères permettant de sélectionner les élèves orientés dans ces classes : là encore le possible étayage de la pédagogie par la psychologie est mis en relief. Marie-Noëlle Allart-Gondouin interroge les conceptions de la culture scolaire comme culture générale et de la culture professionnelle, non seulement à travers les travaux de la commission Langevin-Wallon (qui ne précise pas quels seraient les contenus culturels à privilégier dans les disciplines générales) mais aussi à travers les programmes et une revue professionnelle de l'après-guerre (Technique, art et science). Philippe Mazereau s'intéresse à «la réforme de l'enseignement spécialisé (1962-1967)» qui, là encore, permet de questionner les relations entre psychologie et pédagogie.

7 Il s'agit bien globalement d'un ouvrage d'histoire de l'éducation, qui a le mérite de traiter d'évolutions parfois récentes. Selon leurs questions et leurs objets, les lecteurs y 
trouveront des apports clairs et convaincants et pourront construire des liens suggestifs entre contributions, comme le suggère l'introduction. Toutefois, certains chapitres, tout en traitant d'un type spécifique de savoirs scolaires, outillent le lecteur pour interroger d'autres disciplines ou d'autres contenus. Par exemple, le chapitre rédigé par Joël Lebeaume incite à une lecture curriculaire des changements et des contenus scolaires qui non seulement prenne en compte les programmations d'un même enseignement au fil de la scolarité, et donc les logiques et les tensions de cet ensemble, mais aussi les relations "externes" ou "horizontales", selon ses termes, entre un enseignement et les autres, la conquête d'une spécificité ne s'opérant que par rapport aux autres disciplines, en particulier lorsqu'il s'agit d'installer un nouvel enseignement ou un nouveau contenu. Plusieurs chapitres mettent l'accent sur les jeux entre professionnalité enseignante (avec ce que cela suppose de compétences à innover et de résistances) et projet institutionnel dans la définition des contenus programmés et enseignés, et en particulier sur l'influence des démarches didactiques dans la configuration finale des contenus. Ceci tend à nuancer très fortement les approches qui verraient dans les disciplines scolaires une construction seulement idéologique, même si la question de la culture scolaire pertinente dans et pour la démocratisation de l'enseignement est centrale dans nombre de contributions. Enfin, au-delà des périodes abordées, la lecture de certaines contributions éveille des réflexions très contemporaines : ainsi l'évaluation des capacités intellectuelles des élèves et l'appel à la psychologie ou encore l'influence de l'évaluation des contenus et pratiques structurant la discipline - questions que l'imposition de programmes par compétences a remises au premier plan, tout comme le positionnement des enseignants auxquels est imposée une réforme structurelle des contenus. Les finalités et les mises en pratique de l'enseignement artistique ont aussi fait l'objet de débats renouvelés avec la prescription d'une « histoire des arts » en 2008. D'autres débats sont aussi récurrents : quelle culture scolaire pertinente pour les filières professionnelles? Quelle définition des contenus du «français» pour le primaire ou le secondaire? Quelle part des théories de l'apprentissage dans la formation pédagogique et didactique? Etc. La lecture de cet ouvrage permet donc aussi de prendre du recul ou de donner une épaisseur historique aux débats d'actualité.

\section{AUTEURS}

\section{NICOLE TUTIAUX-GUILLON}

Université de Lille, CIREL-Théodile, ESPE, Comue Lille Nord de France 